\title{
ON THE STATIC ANALYSIS OF ANNULAR RINGH HYDROSTATIC THRUST BEARINGS LUBRICATED WITH COUPLE STRESS FLUIDS
}

\author{
Uday Pratap Singh ${ }^{1}$, Chandra Prakash Singh ${ }^{2}$ \\ ${ }^{I}$ Department of Applied Sciences, Ansal Institute of Technology and Management Lucknow, India \\ ${ }^{2}$ Department of Applied Sciences, Ambalika Institute of Management Technology Lucknow, India
}

\begin{abstract}
In the present theoretical analysis, the static properties of the annular ring hydrostatic thrust bearings have been investigated. The lubricant in the system has been taken as non-Newtonian couple stress fluids. The effects of centrifugal inertia and couple stresses on the film pressure, load capacity and lubricant flow rate have been studied and a good agreement of the present results with the earlier established results has been observed. Analytic solution for pressure distribution has been obtained in the analysis taking into account, the influence of centrifugal inertia in the recess region. The effect of couple stress on pressure was found to be dependent on central recess radius but the decreasing load capacity and flow rate with the increase of couple stress parameter was observed in the analysis. Load capacity was also observed to be higher with the recess radius closer to the axis of the bearing.
\end{abstract}

Keywords: Hydrostatic bearings; annular rings; couple stress fluids; static performance. $* * *$

\section{INTRODUCTION}

Annular hydrostatic thrust bearing is a type of hydrostatic bearing or externally pressurized bearing system. Due to the potential advantages of hydrostatic thrust bearings, several investigations have been presented by the researchers from time to time on design and lubricant effects on the performance of hydrostatic bearing systems [1-3]. The problem of annular hydrostatic thrust bearings has been analysed by Bakker and Ostayen [3] for for Newtonian fluids and Singh et al. [4] for no-Newtonian fluids.

On the other hand, Tribologists have also done a great deal of work to increase the efficiency of stabilizing properties of non-Newtonian lubricants by addition of long chain polymer solutions as viscosity index improvers. The fluids with these improvers have been studied as couple stress fluids [7].

The properties of annular recess hydrostatic thrust bearing were discussed by Bakker and Ostayen [3] and Singh et al. [4] with the inference that most of the lubricants used in the modern industries are not the tradition Newtonian lubricants any more. In the same sequence, a number of microcontinuum models [5-7] have been presented and analysed for various bearings. The effect of the couple stress on hydrostatic step thrust bearings by Gupta and Sharma [8] and Lin [9]. But there are still no studies on the annular recess hydrostatic thrust bearings lubricated with couple stress fluids.

\section{ANALYSIS}

The physical configuration of an externally pressurized annular thrust bearing is shown in figure 1. The lubricant in the system is taken as non-Newtonian couple stress fluid. The body forces and body couples are considered to be absent and the assumptions of thin film lubrications are assumed to be applicable. After Singh et al. [4], the modified Reynolds equation in the present problem is obtained as :

$$
\begin{gathered}
-\frac{\rho \bar{v}^{2}}{\bar{r}}=\frac{\partial \bar{P}}{\partial \bar{r}}+\mu \frac{\partial^{2} \bar{u}}{\partial \bar{z}^{2}}-\eta \frac{\partial^{4} \bar{u}}{\partial \bar{z}^{4}} \\
0=\mu \frac{\partial^{2} \bar{v}}{\partial \bar{z}^{2}}-\eta \frac{\partial^{4} \bar{v}}{\partial \bar{z}^{4}} \\
0=\frac{\partial \bar{P}}{\partial \bar{z}}
\end{gathered}
$$

and the equation of continuity is

$$
\frac{1}{\bar{r}}\left(\frac{\partial \overline{r u}}{\partial \bar{r}}\right)+\frac{\partial \bar{w}}{\partial \bar{z}}=0
$$

To simplify the analysis, the following dimensionless quantities are brought in:

$$
\begin{gathered}
P=\frac{\bar{P}}{P_{o}}, Q=\frac{6 \mu \bar{Q}}{\pi R^{3} P_{o}}, r=\frac{\bar{r}}{R}, r_{o}=\frac{\bar{r}_{o}}{R}, r_{1}=\frac{\overline{r_{1}}}{R}, r_{2}=\frac{\overline{r_{2}}}{R} \\
S=\frac{3 \rho \Omega^{2} R^{2}}{20 P_{o}}, r_{3}=\frac{\overline{r_{3}}}{R}, u=\frac{\bar{u}}{R \Omega}, v=\frac{\bar{v}}{R \Omega}, w=\frac{\bar{w}}{R \Omega} \\
z=\frac{\bar{z}}{R}, \delta=\frac{P_{o}}{\mu \Omega}, \bar{l}=\sqrt{\frac{\eta}{\mu}}, l=\frac{\bar{l}}{R}, h=\frac{\bar{h}}{R}
\end{gathered}
$$


Where $r_{0}$ is the inner radius of the bearing, $\mathrm{R}$ is the outer radius of the bearing, $r_{1}$ is the inner radius of the annular recess, $r_{2}$ is the outer radius of the recess, and $r_{3}$ is the middle radius of the recess. Then $d_{0}$ is the width of the recess, $h$ is the film thickness, and $\beta h$ is the thickness of the recess, and $r_{3}$ is used to decide the location of the recess, while $d_{0}$ is used to describe the scale of the recess. By the geometric relationship showed in fig. 1 , the inner and outer radii of the recess, $r_{1}$ and $r_{2}$, could be expressed by $r_{3}$ and $d_{0}$ as follows:

$$
\begin{aligned}
& r_{1}=r_{3}-\frac{d_{0}}{2} \\
& r_{2}=r_{3}+\frac{d_{0}}{2} \\
& -\frac{S_{0} v^{2}}{r}=-\frac{\partial P}{\partial r}+\frac{1}{\delta} \frac{\partial^{2} u}{\partial z^{2}}-\frac{l^{2}}{\delta} \frac{\partial^{4} u}{\partial z^{4}} \\
& 0=\frac{\partial^{2} v}{\partial z^{2}}-l^{2} \frac{\partial^{4} v}{\partial z^{4}} \\
& 0=\frac{\partial P}{\partial z}
\end{aligned}
$$

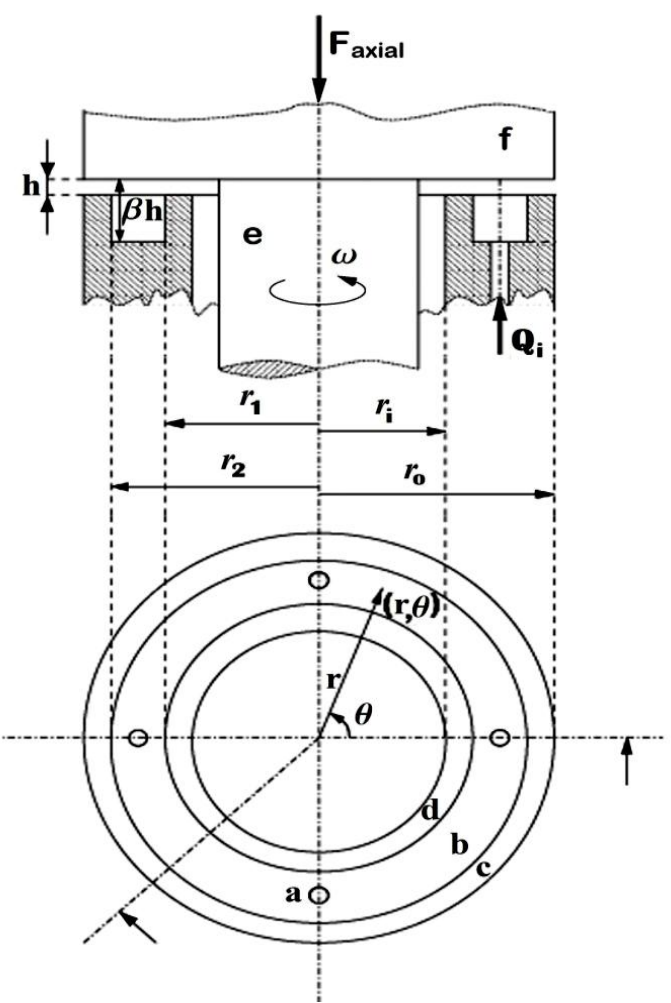

Fig. 1: Schematic diagram of annular recess hydrostatic thrust bearings

$$
\frac{1}{r} \frac{\partial(r u)}{\partial r}+\frac{\partial w}{\partial z}=0
$$

Following Singh [4], the Reynolds equation is obtained as

$$
\begin{gathered}
\frac{1}{r} \frac{\partial}{\partial r}\left(\begin{array}{c}
r\left(\frac{\partial P}{\partial r}-\frac{40 S r l^{2}}{3(\beta+1)^{2} h^{2}}\right) \\
\times f_{1}(\beta h, l)-2 S r^{2} f_{2}(\beta h, l)
\end{array}\right)=0 \\
\begin{aligned}
f_{1}(\beta h, l)= & 24 l^{3} \tanh \left(\frac{(\beta+1) h}{2 l}\right) \\
& +(\beta+1)^{3} h^{3}-12(\beta+1) h l^{2} \\
f_{2}(\beta h, l)= & 40 l^{3} \tanh \left(\frac{(\beta+1) h}{2 l}\right) \\
& +(\beta+1)^{3} h^{3}-20(\beta+1) h l^{2}
\end{aligned}
\end{gathered}
$$

Then integrating the equation (11) with respect to $r$, and the pressure gradient is obtained as

$$
\begin{aligned}
\frac{\partial P}{\partial r}= & \left(\frac{40 l^{2}}{3(\beta+1)^{2} h^{2}}+\frac{2 f_{2}(\beta h, l)}{f_{1}(\beta h, l)}\right) \\
& \times S r+\frac{C_{1}}{f_{1}(\beta h, l)}
\end{aligned}
$$

from which, pressure distribution is obtained as

$$
\begin{aligned}
P(r) & =\left(\frac{20 l^{2}}{3(\beta+1)^{2} h^{2}}+\frac{f_{2}(\beta h, l)}{f_{1}(\beta h, l)}\right) \\
& \times S r^{2}+\frac{C_{1}}{f_{1}(\beta h, l)} \log r+C_{2}
\end{aligned}
$$

The pressure distribution is step by step evaluated in the three regimes of the bearing as follows :

(i) The pressure due to radial flow only

$$
P_{1}(r)= \begin{cases}\frac{b_{1}}{f_{1}(h, l)} \log r+b_{2} ; & r_{o} \leq r \leq r_{1} \\ p_{o} & r_{1} \leq r \leq r_{2} \\ \frac{b_{3}}{f_{1}(h, l)} \log r+b_{4} ; r_{2} \leq r \leq 1\end{cases}
$$


(ii) The pressure due to inertia

$$
P_{2}(r)=\left\{\begin{array}{r}
g(h, l) r^{2}+\frac{c_{1}}{f_{1}(h, l)} \log r+c_{2} ; \\
r_{o} \leq r \leq r_{1} \\
g(\beta h, l) r^{2}+\frac{c_{3}}{f_{1}(\beta h, l)} \log r \\
+c_{4} ; \quad r_{1} \leq r \leq r_{2} \\
g(h, l) r^{2}+\frac{c_{5}}{f_{1}(h, l)} \log r+c_{6} ; \\
r_{2} \leq r \leq 1
\end{array}\right.
$$

where,

$$
\begin{aligned}
c_{1}= & c_{3}=c_{5}=f_{1}(h, l) f(\beta h, l) J \\
c_{2}= & -g(h, l) r_{o}^{2}-f_{1}(\beta h, l) J \cdot \log r_{o} \\
c_{4}= & -g(h, l)\left(r_{2}^{2}-1\right)-g(\beta h, l) r_{2}^{2} \\
& +\left(f_{1}(\beta h, l)-f_{1}(h, l)\right) J \cdot \log r_{2}
\end{aligned}
$$

$c_{6}=-g(h, l)$

$$
J=\frac{\left[\begin{array}{l}
g(\beta h, l)\left(r_{1}^{2}-r_{2}^{2}\right) \\
-g(h, l)\left(1-r_{o}^{2}+r_{1}^{2}-r_{2}^{2}\right)
\end{array}\right]}{\left[\begin{array}{l}
f_{1}(\beta h, l)\left(\log r_{1}-\log r_{o}-\log r_{2}\right) \\
-f_{1}(h, l)\left(\log r_{1}-\log r_{2}\right)
\end{array}\right]}
$$

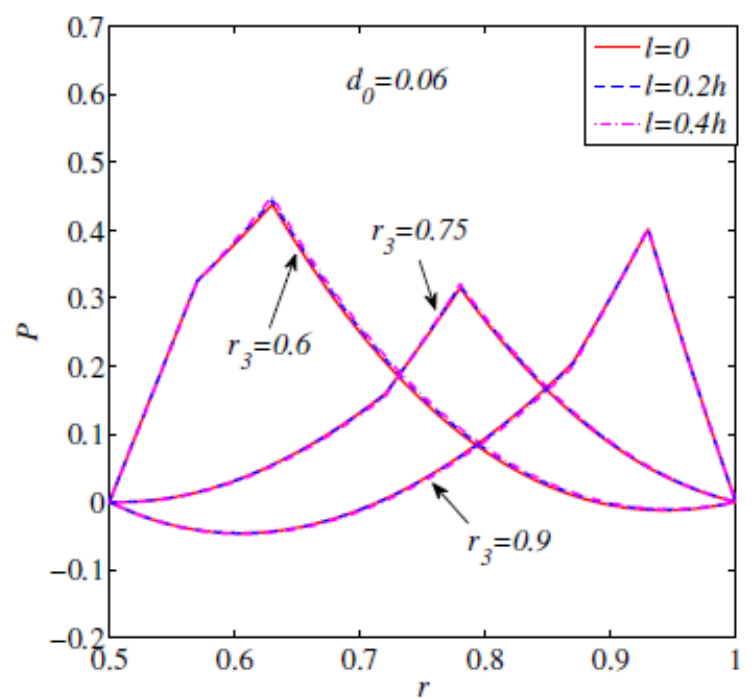

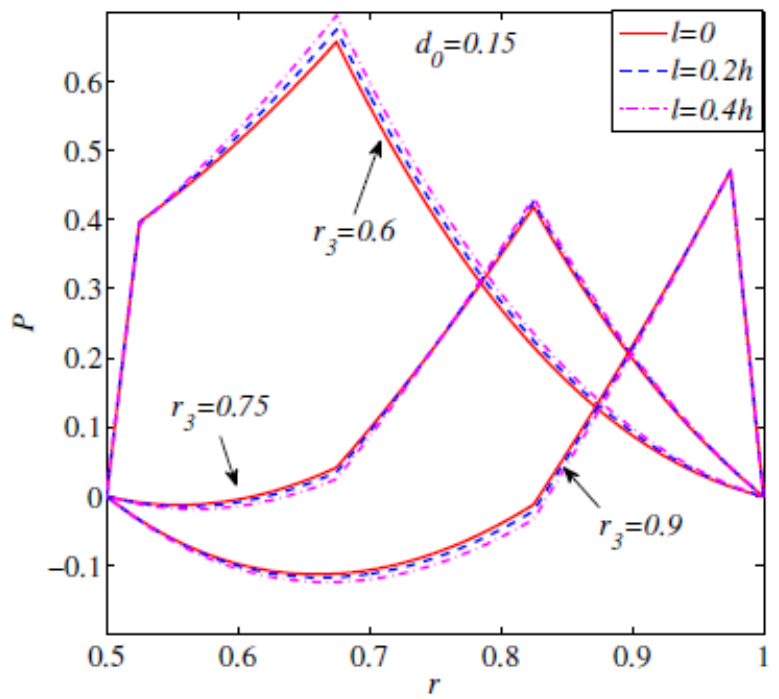

Fig. 2: Pressure distributions of various bearing structures lubricated with different couple stress fluids.

\section{RESULTS AND DISCUSSION}

To discuss the static properties of hydrostatic thrust bearings with annular recess lubricated by couple stress fluids, the results of pressure distribution, flow rate and load capacity are presented with the parameters $d_{1}=1-r_{0}, d_{2}=\left(1+r_{0}\right) / 2$; Singh[4].
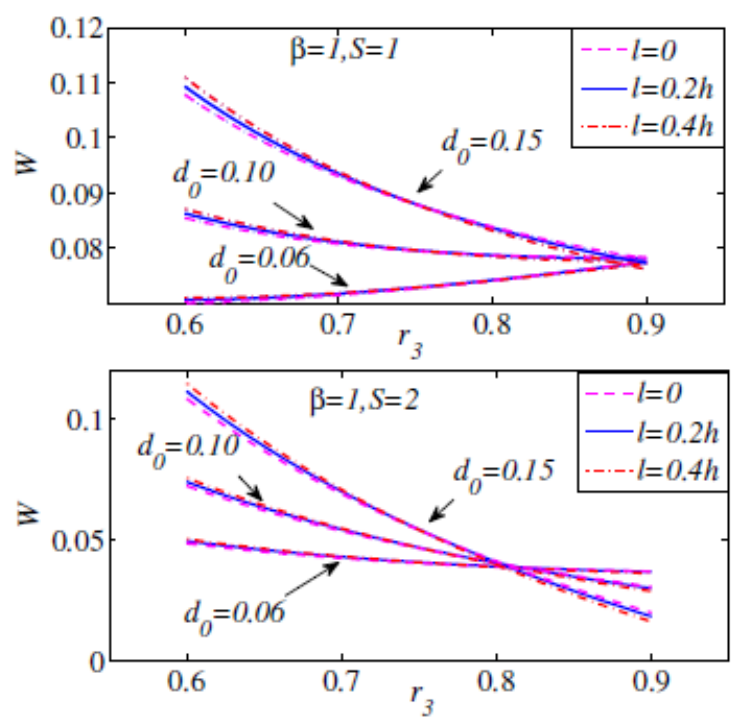

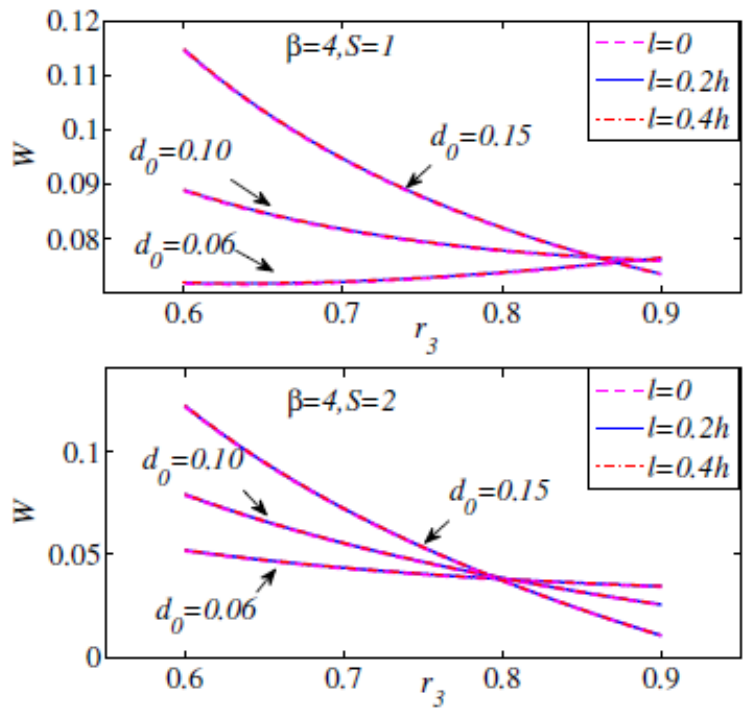

Fig.3: Variations of load capacity with respect to recess centre radius. $\left(r_{0}=0.5\right)$

The effect of the bearing structure is showed in fig. 2. The inner radius of the bearing is $r_{0}=0.5$, depth of annular recess is $\beta=1$, the inertia coefficient $S=2$, the couple stress parameter $l=0,0.2 h, 0.4 h$, the middle radius of the recess is $r_{3}=0.6,0.75,0.9$, and the recess width is: $(a) d_{0}=0.06 ;(b)$ $d_{0}=0.15$. When $d_{0}=0.06$, no matter where the recess lies, the effect of the couple stress is negligible. For $d_{0}=0.15$, when the middle radius of the recess is coincidence with the middle radius of the bearing $\left(r_{3}=\left(r_{0}+1\right) / 2=0.75\right)$, the effect of the couple stress is also negligible. While the recess middle radius differs from the bearing middle radius $\left(r_{3}=\right.$ $0.6,0.9)$, the effect of the couple stress is gradually evident. No matter some part between $r=r_{2}$ and the bearing outer radius $(r=1)$ could be negative. To avoid this situation, during the process of designing the thrust bearing, the minimum pressure between $r=r_{2}$ and the bearing outer radius $(r=1)$ should be positive. So differentiates the expression of $P$ on $r_{2}<r<1$ with respect to $r$, and makes the result less than zero, then inequality what the bearing structure is, the location of the maximum pressure is always $r$ $=r_{2}$.

Fig. 3 show the variations of load capacity with respect to recess radius $r_{3}$. The effect of the couple stress on load capacity is found negligible. The increases of the recess depth and the inertia effect slightly enhance load capacity. And with a wider recess width, the bearing load capacity would be enhanced notable. In general, when the location of the recess is closer to the bearing inner radius, the bearing load capacity is better.

\section{CONCLUSION}

The combined effects of inertia, bearing structure and couple stress on the static properties (pressure, flow rate and load capacity) of annular recess hydrostatic thrust bearing are studied. The effects of the inertia and couple stress on pressure distribution are slight, and the pressure distribution is mainly decided by the bearing structure. When the radius of the recess is closer to the bearing inner radius, or the width of the recess is wider, the better pressure distribution would be obtained.

\section{REFERENCES}

[1] Dowson, D. (1961). Inertia effects in hydrostatic thrust bearings. J Fluid Eng, 83(2), 227-234.

[2] Coombs, J. A., and Dowson, D. (1964, June). Paper 12: An Experimental Investigation of the Effects of Lubricant Inertia in a Hydrostatic Thrust Bearing. In Proceedings of the Institution of Mechanical Engineers, Conference Proceedings (Vol. 179, No. 10, pp. 96-114). SAGE Publications.

[3] Bakker, O. J., and Van Ostayen, R. A. J. (2010). Recess depth optimization for rotating, annular, and circular recess hydrostatic thrust bearings. J Tribol, 132(1), 011103.

[4] Singh, U. P., Gupta, R. S., and Kapur, V. K. (2013). On the application of Rabinowitsch fluid model on an annular ring hydrostatic thrust bearing. Tribol Int, 58, 65-70.

[5] Ariman, T. T. N. D., Turk, M. A., and Sylvester, N. D. (1974). Applications of microcontinuum fluid mechanics. Int J Eng Sci, 12(4), 273-293.

[6] Ariman, T. M. A. N. D., Turk, M. A., and Sylvester, N. D. (1973). Microcontinuum fluid mechanics-a review. Int J Eng Sci, 11(8), 905-930.

[7] Stokes, V. K. (1966). Couple stresses in fluids. Phys Fluids (1958-1988), 9(9), 1709-1715.

[8] Gupta, R. S., and Sharma, L. G. (1988). Analysis of couple stress lubricant in hydrostatic thrust bearing. Wear, 125(3), 257-269.

[9] Lin, J. R. (1999). Static and dynamic characteristics of externally pressurized circular step thrust bearings lubricated with couple stress fluids. Tribol Int, 32(4), 207-216.

[10] Singh, U. P., Gupta, R. S., and Kapur, V. K. (2011). On the steady performance of hydrostatic thrust bearing: Rabinowitsch fluid model. Tribol T, 54(5), 723-729. 